\title{
System for detection and analysis of partial discharges under transient voltage application
}

\author{
Søren Valdemar Kjær \\ DONG Energy Wind Power A/S \\ Denmark
}

\begin{abstract}
A measuring system for detection and analysis of partial discharges (PD) in electrical insulation under fast repetitive pulse voltage application has been developed. Partial discharges can be triggered by transient voltages and are considered as being one initial cause of insulation failure.

The experimental setup consists of a specially designed pulse generator for generation of fast voltage transients. The pulses are unipolar, with the peak variable voltage of up to $10 \mathrm{kV}$ and with an adjustable frequency from 0 $\mathrm{Hz}$ to $1.7 \mathrm{kHz}$, burst frequency of up to $2 \mathrm{MHz}$.

Partial discharge inception under pulse voltage application was determined by data acquisition by means of a standard oscilloscope and suitable data analyses in Matlab, using wavelet transform and denoising techniques. The measurement results are presented in a partial discharge classification pattern designed to be used in connection with fast repetitive pulses as originating from power electronics.

The feasibility of the test system for insulation diagnostics was illustrated by application to twisted pairs of enamelled winding wire.

The test system is suitable for achieving new results to get better knowledge of degradation mechanisms in insulating materials exposed to fast transients from eg power electronics.
\end{abstract}

\section{Introduction}

It is well known that the wide use of power electronic converters is one of the causes of current and voltage distortion in the electrical networks. Part of these distortions are repetitive pulses with high slew rates (level of voltage change/time). Significant reduction of the reliability of insulation systems experiencing distorted voltage/current waveforms has been observed in the AC power system network. Two aging processes are dominating with respect to electrical insulation degradation under impulse voltages, energy losses dissipated in the dielectric and partial discharges (PD). PD should be considered when evaluating insulation material performance, even though it has been proved [1] that aging is accelerated due to repetitive voltage pulses, even in the absence of PD. PD are often considered as the initial stage of the final insulation

\author{
Joachim Holbøll \\ Technical University of Denmark \\ Denmark
}

failure and can be triggered by over voltages in general, which also includes transient over voltages.

Investigation of how different solid insulating materials deteriorate under fast repetitive voltage pulses involves developing tools for voltage application and for measurement of the materials response. In this case, the experimental setup consists of test object, pulse generator, PD measurement system, data collection and analysis tools.

\section{Test Setup}

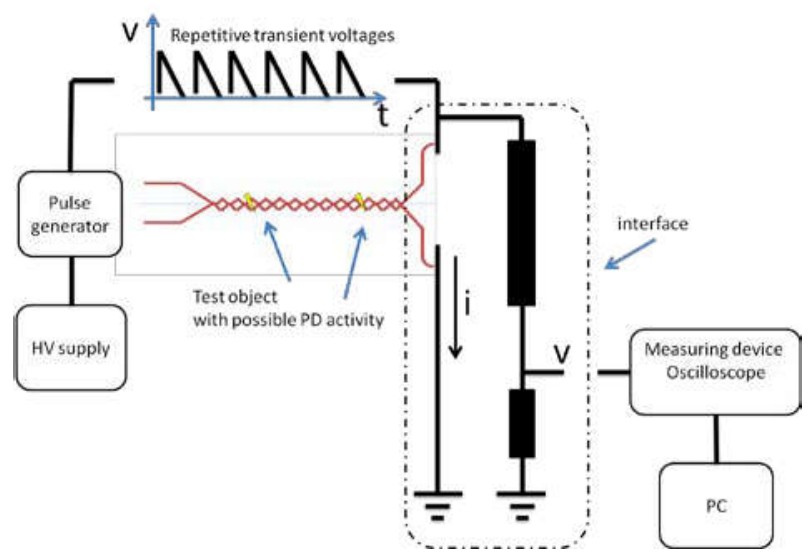

Figure 1: Test setup with twisted wire test object, pulse generator and measuring system

The test setup is seen in figure 1 and consists of the following main parts:

The test object with the insulation material exposed to repetitive voltage pulses, resulting in $\mathrm{PD}$ activity. The test object can be compared to a capacitor and the current flowing through it can be measured. It is clear from the figure that the current caused by the applied voltage pulse is superimposed to possible PD-currents from the test object, which we wish to measure.

The test object is, as defined in IEC 60505, either the component desired to be tested or an object in which there is sufficient similarity between the object and the component desired to be tested. In this project the test specimens are twisted pairs of two enamelled wires wounded in a standard way, hereby giving conditions comparable to an electrical machine. 
Preparation of these test objects is outlined in the IEC 172 : 1987, Test procedure for the determination of the temperature index of enameled winding wires.

A high voltage impulse generator, capable of generating fast repetitive voltage pulses.

In order to investigate the consequence of repetitive transient voltages, in principle all types of transient voltages should be considered under repetitive conditions. We therefore need a pulse generator capable of generating repetitive high voltage pulses, with the following characteristics to be adjustable in a range as broad as possible:

- Peak voltage

- Rise time/Slew rate

- Repetition rate.

The complexity of the pulse generator design is primarily dependent on the size of the peak voltage desired.

It is rather easy to get hold of resistors and capacitors that can handle high voltage, however getting hold of a switch with the ability to rapidly switch high voltages (high currents) at very high speeds, is far more requiring.

A solid state switch here was an obvious choice, since it is superior to the other switches in particular in terms of switching frequency, better controllability and no contact bounce. Figure 2 shows the switch chosen for the test setup.

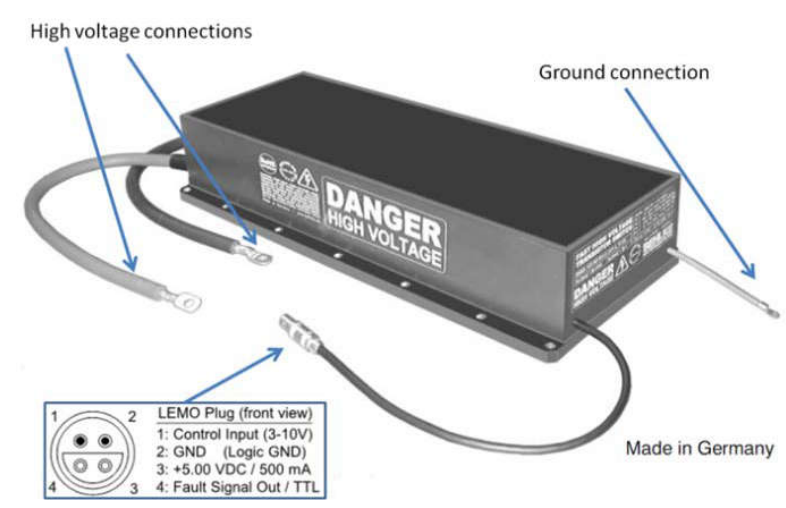

Figure 2: High voltage switch (Behlke HTS 501-10-LC2)

The pulse generator is controlled by a function generator which triggers a HP 8082A Pulse generator (0-5V), which provides the input to Behlke solid state switch.

A measurement system, which is able to measure the current changes (PD activity) in the test object, during fast voltage transients. The measurement system includes interface, measuring device, digital signal processing and presentation:
- the interface between the test object the measuring device, is a high frequency current transformer (HFCT) more specifically a Rohde \& Schwarz, Model 02: $20 \mathrm{~Hz}$ to $100 \mathrm{MHz}$,EZ-17. A high voltage probes allows for simultaneous voltage measurements.

- the measuring device is a Tektronix TDS2014B $100 \mathrm{MHz}$ Digital Oscilloscope. The oscilloscope has a record length of 2500 points at all time-bases, this has influence on the number of decomposition levels, in combination with the mother wavelet, which can be used in the wavelet transform.

- data connection between the oscilloscope and a PC. Matlab is installed on the PC and the Matlab code creates a Virtual Instrument Software Architecture (VISA) USB object, a device object and connects the device object to hardware (oscilloscope). This allows the oscilloscope to be controlled from matlab. The data connection is relatively slow. The time between each measurement is approx. 3 seconds which is a major limitation in the measuring system.

\section{PD detection during transients}

The major challenge is to detect PD under transients which in time are in the same range as the PD pulses.

Figure 3 shows an example of how the superposition of the applied voltage pulse and the PD activity inside the test object looks like, seen from the measuring device.

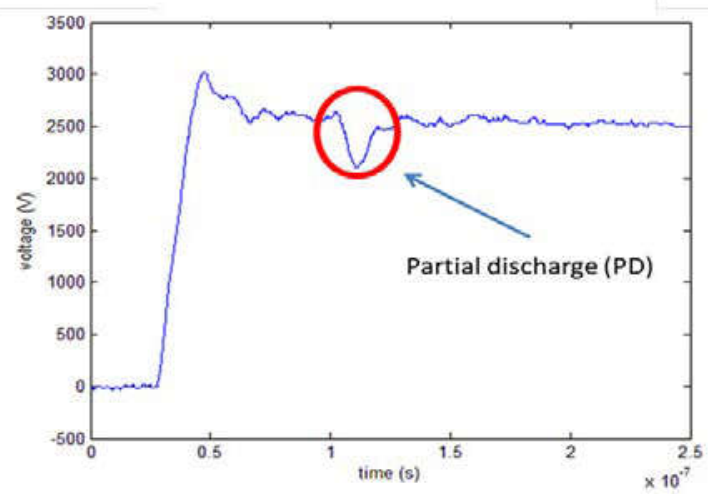

Figure 3: Voltage measurement

It is seen that the voltage pulse rises rapidly from 0 to $2.5 \mathrm{kV}$, with an overshoot of about $500 \mathrm{~V}$. Approx $50 \mathrm{~ns}$ after the voltage has reached $2.5 \mathrm{kV}$, we see a sudden drop in voltage that quickly restores its original value. The sudden drop in voltage is caused by PD activity, formation and the redistribution of the charges within the test object. 


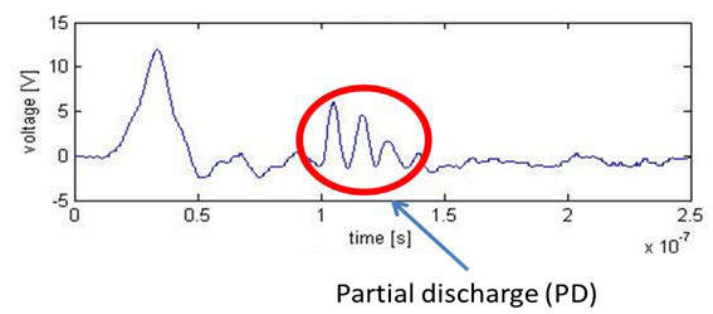

Figure 4: Current measurement

Figure 4 shows measurement of the current that flows through the test object, measured between the test object and ground. Compared to the PD voltage pulse, more oscillations can be seen.

The main task is to develop a system that automatically detects PD pulses and separates them from the applied voltage/current pulse.

\section{Signal analysis traditional vs wavelet analysis}

Digital signal processing is often conducted in one of the following domains: frequency domain, time domain, correlation domain or wavelet domain.

A wavelet is a small wave with a limited duration and which has an average value of zero. In contrast to the sinusoids which theoretically extend from minus to plus infinity, wavelets have a beginning and an end.

When explaining what a wavelet is, it is natural to compare it to a transient. Signals lasting for a very short period of time are referred to as a transient signal. In our case, $\mathrm{PD}$ is comparable to transients, and therefore it is natural to use wavelet transformation to digital signal processing of PDs [2, 3].

Like the discrete Fourier transform breaks a signal into sinusoids, the discrete wavelet transform breaks a signal into mentioned small waves, wavelets. These waves can then be added together to reform the signal [4].

In this study, the Wavelet transform was chosen for the following reasons [5]:

Wavelet transform is a time-frequency analysis meaning that it studies a signal in both the time and frequency domains simultaneously.

The advantage of the wavelet domain over the frequency domain is that it captures both frequency and location information. This means that although the frequency content of the applied voltage pulse is comparable with the frequency content of PD, the wavelet transform will still be able to separate the two parts of the signal from each other.

Wavelet transform has advantages over traditional Fourier methods in analysing physical situations where the signal contains discontinuities and sharp spikes (like PD). In a way, the wavelet domain, the benefits from the other domains; frequency domain, the time domain, and the correlation domain, because it offers the opportunity to represent the signal under analysis in both the time and frequency domain, and in a way, as we shall see it does so, using the cross-correlation technique.

\section{PD analysis by means of wavelets}

The following figure shows decomposition of the PD signal and consists of 10 plots. At the top the original signal is sampled and recorded by the oscilloscope. The second plot, from the top, shows the approximation coefficients at level 8 (a8). The eight next plots show the detail coefficients from level 8 (d8) to level 1 (d1).

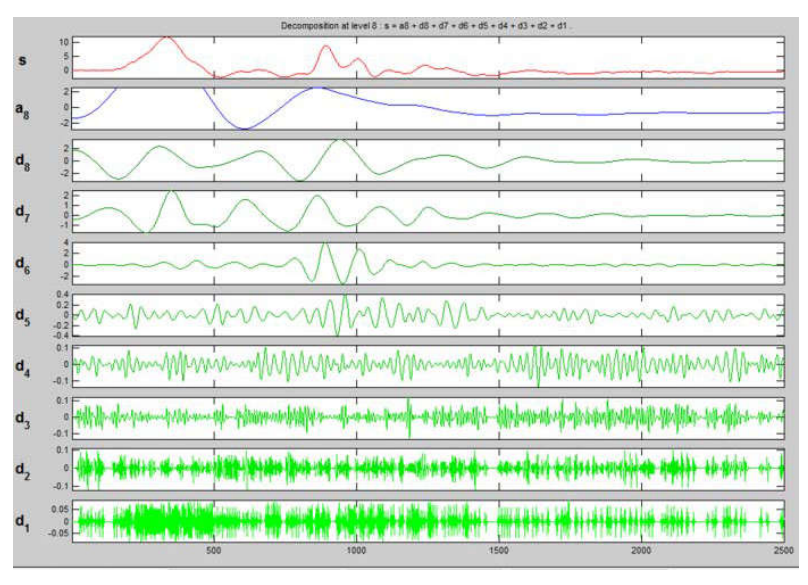

Figure 5: PD detection on the current measurement by decomposition at level 8 using wavelet $\mathrm{db8}$

It is seen that at decomposition level $\mathrm{d} 6$ the wavelet $\mathrm{db} 8$ generates the largest coefficients for the PD pulse and the coefficients are positioned very precisely around the PD pulse and no large coefficients are seen at the flank.

It is now relatively easy to extract information about:

- the position of the flank

- the position of the PD pulse

- the size of the PD pulse.

\section{Representation of the results}

With the information we may extract out of the current measurements, it is possible to generate some kind of fingerprints for the partial discharge measurements, and test objects. The characteristic fingerprint is often referred to as a PD pattern of the measurement. During aging it is expected that it will be possible to observe significant changes in the PD patterns during these tests.

Using cluster analysis techniques, different groups of PD patterns obtained during aging can be discriminated and a data base of the patterns can be created. The data base can potentially be used to characterize different stages in the aging process.

Let us consider an accumulation of actual current measurements, figure 6 . 


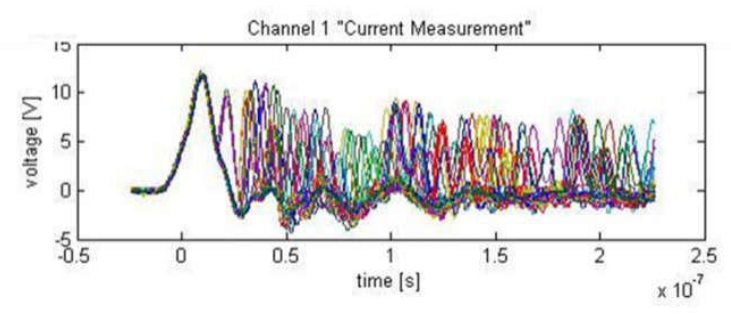

Figure 6: Accumulation of actual current measurements

The figure clearly shows the varying position of PD occurrence and some different amplitude. Using the wavelet-based analysing tool we see a distribution as shown in figure 7.

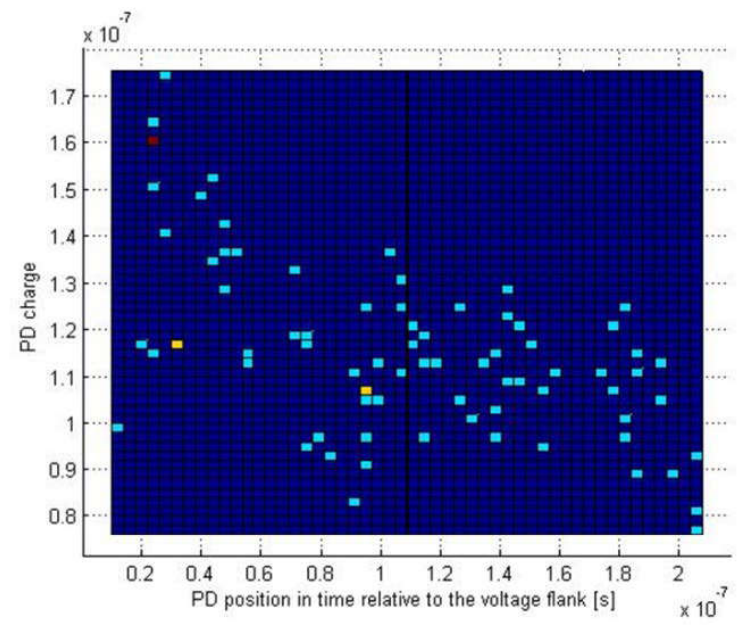

Figure 7: Plot of PD position, PD charge and number of discharges

The colours in the plot indicate the number of discharges/dot. With the few measurements here $(<100)$ there is almost only 1 detection/dot.

\section{Discussion}

It is clear that discharges are easily identified and that the wavelet-based algorithm works and fulfils the expectations.

A first analysis of the results indicates a tendency towards largest discharges occurring closest to the front of the applied voltage pulse.

The results also showed that just around the PD inception voltage the positions of the PDs are spread out over a larger span of time relative to the voltage flank.

Further investigations are of course necessary to systematically investigate the discharges behaviour and the resulting pattern as a function of:
- $\quad$ voltage level

- repetition frequency

- $\quad$ applied pulse rise time

- type of test object.

\section{Conclusion}

A measuring system has been constructed able to generate, detect, analyse and classify partial discharge under transient voltage application.

A pulse generator, which is capable of generating fast repetitive high voltage pulse, has been designed suitable for tests with controlled peak voltage, variable pulse rise time and repetition rate.

A detection system has been developed capable of detecting PD pulses under fast repetitive voltages flanks, using a current probe and a voltage probe as an interface between the physical test setup and the measurement device. Signal and data processing is carried out in Matlab by means of wavelet transform.

For presentation of the results of the measurements, a PD pattern suitable for classification of fast repetitive pulses has been developed.

\section{References}

[1] Stone, G.C., R.G. van Heeswijk, and R. Bartnikas, Investigation of the effect of repetitive voltage surges on epoxy insulation. Energy Conversion, IEEE Transactions on, 1992. 7(4): p. 754-760.

[2] D. Lee Fugal, Conceptual Wavelets in Digital Signal Processing, Space \& Signals Technologies LLC, www.ConceptualWavelets.com, 2009.

[3] X. Ma, C. Zhou, and I.J. Kemp, Automated Wavelet Selection and Thresh-olding for PD Detection, IEEE Electrical Insulation Magazine, DEIS Fea-ture article, March/April, Vol. 18, No. 2 2002.

[4] M. Weeks, Digital Signal Processing Using MATLAB and Wavelets, ISBN: 0-9778582-0OInfinity Science Press LLC, 11 Leavitt Street Hingham, MA 020432007.

[5] X. Ma, C. Zhou and I. J. Kemp, Interpretation of Wavelet Analysis and Its Application in Partial Discharge Detection, IEEE Transactions on DielecIrics and Electrical Insulation Vol. 9, No. 3, June 2002. 